\title{
Calculation of the Density of Electrolyte Solution
}

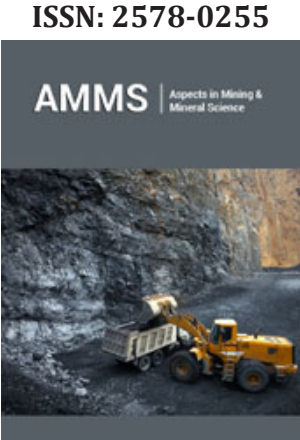

*Corresponding author: GR Allakhverdov, State Scientific Research Institute of Chemical Reagents and High Purity Chemical Substances-National Research Centre Kurchatov's Institute, Russia

Submission: 眥 August 12, 2019

Published: 眥 September 04, 2019

Volume 3 - Issue 3

How to cite this article: Allakhverdov G, Zhdanovich O. Calculation of the Density of Electrolyte Solution. Aspects Min Miner Sci.3(3). AMMS.000565.2019.

DOI: 10.31031/AMMS.2019.03.000565

Copyright@ GR Allakhverdov, This article is distributed under the terms of the Creative Commons Attribution 4.0 International License, which permits unrestricted use and redistribution provided that the original author and source are credited.

\section{Allakhverdov GR* and Zhdanovich $\mathrm{OA}$}

State Scientific Research Institute of Chemical Reagents and High Purity Chemical SubstancesNational Research Centre Kurchatov's Institute, Russia

\section{Abstract}

Based on the solvate model of solutions, the relation of density with the thermodynamics functions is established and an equation for the density of binary solutions is obtained. A generalization of this equation to mixed electrolyte solutions is given.

Keywords: Partial molar volume; Activity of solvent; Hydration number

\section{Introduction}

The density of electrolyte solutions plays a large role in the processing mineral row materials, since in many cases it is the main source of information of the concentration of substances in solutions. The exact equation for the density of solutions is also of great theoretical importance for determining the partial molar volumes of electrolytes and the osmotic pressure of solutions. This article proposes a method for describing the density of binary and mixed solutions on the solvate model of solutions.

\section{Theory}

Let us consider a binary solution containing m moles of electrolyte and w mole solvent (e.g. water) in the volume of solution V. If we assume, without loss of generality, that positive and negative ions form hydrated complexes of a fixed composition containing $n_{p}$ and $n_{n}$ solvent molecules, respectively. than the volume of such solution can be determine as

$$
V=N_{w}^{*} V_{w}+N_{p}^{*} V_{p}+N_{n}^{*} V_{n}+N_{h p}^{*} V_{h p}+N_{h n h n}^{*} V_{h n}
$$

where $\mathrm{N}_{\mathrm{w}}{ }^{*}, \mathrm{~N}_{\mathrm{p}}{ }^{*}, \mathrm{~N}_{\mathrm{n}}{ }^{*}, \mathrm{~N}_{\mathrm{hp}}{ }^{*}, \mathrm{~N}_{\mathrm{hn}}{ }^{*}$ are equilibrium amounts of solvent, ions and hydrated complexes, $\mathrm{V}_{\mathrm{w}}$ is molar volume of pure solvent, $\mathrm{V}_{\mathrm{p}}, \mathrm{V}_{\mathrm{n}}, \mathrm{V}_{\mathrm{hp}}, \mathrm{V}_{\mathrm{hn}}$ are molar volumes of ions and hydrated complexes, respectively. Taking into account the equations of material balance

$$
\begin{gathered}
v_{p} m=N_{p}^{*}+N_{h p}^{*}, \\
v_{n} m=N_{n}^{*}+N_{h n}^{*}, \\
w=N_{w}^{*}+n_{p} N_{h p}^{*}+n_{n} N_{h n}^{*},
\end{gathered}
$$

Eq.(1) can be represented in the form

$$
V=w V_{w}+v_{p} m V_{p}+v_{n} m V_{n}+N_{h p}^{*} \Delta V_{p}+N_{h n}^{*} \Delta V_{n}(2)
$$

where $v_{\mathrm{p}}$ and $v_{\mathrm{n}}$ are the stoichiometric coefficients $\left(v=v_{\mathrm{p}}+v_{\mathrm{n}}\right.$ is stoichiometric coefficient of electrolyte), $\Delta V_{p}=V_{p}+h_{p} V_{w}-V_{h p}, \Delta V_{n}=V_{n}+h_{n} V_{w}-V_{h n}$. Here the hydration number of corresponding current concentration of each ions $h_{\mathrm{p}}, \mathrm{h}_{\mathrm{n}}$ can be defined as

$$
h_{p}=\frac{n_{p} N_{h p}^{*}}{v_{p} m} \quad h_{n}=\frac{n_{n} N_{h n}^{*}}{v_{n} m} \text { (3) }
$$

These values according to Ref.[1] can be expressed in the form 


$$
h_{p}=\frac{n_{p} k_{p} a^{n} p_{w}}{1+n_{p} k_{p} a^{n}{ }^{n}{ }_{w}}, \quad h_{n}=\frac{n_{n} k_{n} a^{n_{n}}{ }_{w}}{1+n_{n} k_{n} a^{n_{n}}{ }_{w}}
$$

where $K_{p}$ and $K_{n}$ is constant of formation of hydrated complex, $a_{w}$ is the activity of solvent.

Using Eq. (3), Eq. (2) can be transformed to

$$
V=w V_{w}+\sum v_{i} m V_{i}-\sum v_{i} m \frac{h_{i}}{n_{i}} \Delta V_{i}(5)
$$

Differentiating Eq. (5), we can determine the partial molar volume of the electrolyte, which in the infinitely dilute solution takes on the value

$$
v_{e}^{0}=\lim _{m \rightarrow 0} \frac{\partial V}{\partial m}=\sum v_{i} V_{i}-\sum v_{i} \frac{h_{i}^{0}}{n_{i}} \Delta V
$$

Combining Eq. (5) and Eq. (6), we have

$$
V=w V_{w}\left(1+\frac{m v_{e}^{0}}{w} V_{w}-\frac{m}{w} \sum v_{i} \frac{h_{i}-h_{i}^{0}}{n_{i}} \frac{\Delta V_{i}}{V_{w}}\right) \text { (7) }
$$

where $h_{i}{ }^{0}$ is the hydration number of ions in the infinitely dilute solution, which can be determined from Eq. (4) at $a_{w} \rightarrow \rightarrow 1$. The density of the solution is determined by the formula

$$
\rho=\frac{M_{e} m+M_{w} w}{V}(8)
$$

where $\mathrm{M}_{\mathrm{e}}$ and $\mathrm{M}_{\mathrm{w}}$ are molecular weights of the electrolyte and solvent, respectively. Mass fraction of electrolyte in solution z can be defined as

$$
z=\frac{M_{e} m}{M_{e} m+M_{w} w}
$$

hence the equality

$$
\frac{m}{w}=\frac{M_{w}}{M_{e}} \frac{z}{1-z}
$$

Substituting Eq. (10) into Eq. (7) and then the result obtained into Eq. (8), the latter can be expressed as

$$
\frac{1}{\rho}=\frac{1}{\rho_{w}}\left(1-z+z \frac{\rho_{w} v_{e}^{0}}{M_{e}}-z \frac{M_{w}}{M_{e}} \sum v_{i} \frac{h_{i}-h^{0}{ }_{i}}{n_{i}} \frac{\Delta V_{i}}{V_{w}}\right)
$$

where $\rho_{\mathrm{w}}$ is the density of pure solvent. Let us further imagine the hydration number of the electrolyte $h_{i}$ as a function of the concentration z. The activity of the solvent according [1] can be determined using Eq. (10) as

$$
\ln a_{w}=-v \varphi \frac{m}{w}=-v \varphi \frac{M_{w}}{M_{e}} \frac{z}{1-z}
$$

where $\varphi$ is the osmotic coefficient of the solution. Using Eq. (4), the value of $h_{i}$ can be expanded in a Taylor series and limited to a linear term to represent it in the form

$$
h_{i}=h_{i}^{0}+\left(\frac{d h_{i}}{d \ln a_{w}} \frac{d \ln a_{w}}{d z}\right)_{0} z=h_{i}^{0}-\frac{v n_{i} h_{i}^{0}}{1+k_{i}} \frac{M_{w}}{M_{e}} z
$$

Substituting Eq. (13) into Eq. (11) we finally have

$$
\frac{1}{\rho}=\frac{1}{\rho_{w}}\left(1-A z+B z^{2}\right)
$$

$$
\text { where } A=1-\frac{\rho_{w} v_{c}^{0}}{M_{e}}, B=v \frac{M_{w}^{2}}{M_{e}^{2}} \sum \frac{v_{i} h_{i}^{0}}{1+k_{i}} \frac{\Delta V_{i}}{V_{w}} \text {. }
$$

Next, we use the relationship of dimensionless concentration $\mathrm{z}$ with molality c. Combining equality c=m/V and Eq. (8), (9) we have

$$
z=\frac{M_{e}}{\rho} c(15)
$$

Substituting Eq. (15) into Eq. (14) and limiting to the quadratic term, we can determine the density of solution in the form

$$
\rho=\rho_{w}+a c-\frac{b c^{2}}{\rho_{w}+a c}(16)
$$

where $a=M_{e} A, b=M_{e}{ }^{2} B$.

Eq. (14) can be extended to mixed electrolyte solutions. If we neglect the influence of the last term Eq. (14), the density of the mixed solution can be calculated by the principle of additivity and is presented in the form

$$
\frac{1}{\rho}=\frac{1}{\rho_{w}}\left(1-\sum A_{i} z_{i}+\left(\sum z_{i}\right) \sum B_{i} z_{i}\right)
$$

Further, using Eq. (15), (16), the density of the mixed solution can be expressed by molality

$$
\rho=\rho_{w}+\sum a_{i} c_{i}-\frac{\sum M_{e i} c_{i}}{\rho_{w}+\sum a_{i} c_{i}} \sum \frac{b_{i} c_{i}}{M_{e i}}
$$

\section{Discussion}

Table 1 shows the applicability of the model used for electrolytes

\begin{tabular}{|c|c|c|c|c|c|}
\hline Electrolyte & $\mathrm{a}, \mathrm{kg} / \mathrm{mol}$ & b, $\mathrm{kg}^{2} / \mathrm{mol}^{2}$ & $\mathrm{v}_{\mathrm{c}}^{0}, \mathrm{~cm}^{3} / \mathrm{mol}$ & $\Delta, \%$ mass & $\delta, \%$ \\
\hline $\mathrm{HCl}$ & 0,0180 & 0,0003 & 18,6 & $1-40$ & 0,02 \\
\hline $\mathrm{LiOH}$ & 0,0279 & 0,0011 & $-4,0$ & $1-10$ & 0,02 \\
\hline $\mathrm{LiNO}_{3}{ }^{*}{ }^{3}$ & 0,0390 & 0,0002 & 30,0 & $1-40$ & 0,02 \\
\hline $\left.\operatorname{Lil}^{*}\right)^{2}$ & 0,0972 & 0 & 36,7 & $1-60$ & 0,02 \\
\hline
\end{tabular}
of various types. And in all cases, the main contribution of the linear term of Eq. (17) from which it is possible to calculate the partial molar volume of the electrolyte. To estimate this value, we present the volume of the hydrated complex as the sum of the volumes of the central ion and the surrounding water molecules where per one molecule of water accounts for a significantly smaller volume $\mathrm{V}_{\mathrm{w}}{ }^{*}$, then in structure of pure water $\mathrm{V}_{\mathrm{w}}$. Then $V_{h i}=V_{i}+h_{i} V_{w}$ and the volume of solution can be represented as

$$
V=w V_{w}+m \sum v_{i} V_{i}-m \sum v_{i} h_{i}\left(V_{w}-V_{w}^{*}\right)
$$

and further partial molar volume as

$$
v_{c}=\sum v_{i} V_{i}-\sum v_{i} h_{i}\left(V_{w}-V^{*}{ }_{w}\right)
$$

Table 1: The parameters of equation of aqueous solution (16) at $298 \mathrm{~K} .{ }^{*}$ ) at $298 \mathrm{~K} ; \Delta$ is interval of concentration; $\delta$ is average approximation error; calculation according experimental data Ref. [3, 4]. 


\begin{tabular}{|c|c|c|c|c|c|}
\hline $\mathrm{NaCl}$ & 0,0409 & 0,0008 & 17,5 & $1-26$ & 0,02 \\
\hline $\mathrm{NaNO}_{3}{ }^{*}$ & 0,0551 & 0,0009 & 30,1 & $1-45$ & 0,02 \\
\hline $\mathrm{KBr}$ & 0,0846 & 0,0009 & 34,5 & $1-40$ & 0,01 \\
\hline $\left.\mathrm{KI}^{*}\right)$ & 0,1192 & 0,0007 & 46,9 & $1-60$ & 0,02 \\
\hline $\mathrm{RbI}$ & 0,1620 & 0,0012 & 50,4 & $1-60$ & 0,03 \\
\hline $\mathrm{Cs}_{2} \mathrm{SO}_{4}$ & 0,2982 & 0,0085 & 63,8 & $1-26$ & 0,05 \\
\hline $\mathrm{MgCl}_{2}$ & 0,0777 & 0,0028 & 17,6 & $2-32$ & 0,02 \\
\hline $\mathrm{MgSO}_{4}$ & 0,1201 & 0,0065 & 0,3 & $2-26$ & 0,02 \\
\hline $\mathrm{Mn}\left(\mathrm{NO}_{3}\right)_{2}$ & 0,1379 & 0,0046 & 41,1 & $2-55$ & 0,04 \\
\hline $\mathrm{CuCl}_{2}$ & 0,1239 & 0,0057 & 10,6 & $1-26$ & 0,03 \\
\hline $\mathrm{CuSO}_{4}$ & 0,1626 & 0,0089 & $-3,0$ & $1-18$ & 0,02 \\
\hline $\mathrm{ZnSO}_{4}$ & 0,1645 & 0,0089 & $-3,1$ & $2-30$ & 0,02 \\
\hline $\mathrm{CdSO}_{4}$ & 0,2009 & 0,0085 & 7,6 & $2-40$ & 0,03 \\
\hline $\mathrm{FeCl}_{3}$ & 0,1335 & 0,0057 & 28,7 & $1-50$ & 0,05 \\
\hline $\mathrm{LaCl}_{3}$ & 0,2254 & 0,0095 & 19,9 & $1-40$ & 0,02 \\
\hline
\end{tabular}

It is obvious that at high values of the difference $\left(V_{w}-V_{w}^{*}\right)$ the partial molar volume can even have negative values. Thus, this volume reflects structural change in the solution. Eq. (16) can be compared with the widely used Root equation [2].

$$
\rho=\rho_{w}+a^{\prime} c+b^{\prime} c^{2}
$$

where the parameter a' completely corresponds to the parameter a: $a^{\prime}=M_{e}-\rho_{w} v_{c}^{0}$. Hence, we can for each equation determined partial molar volume and compared these values with the experimental data (Table 2). For this purpose, we use Eq.(8) from where follows

$$
v_{c}=\frac{\partial V}{\partial m}=\frac{M_{e}}{\rho}-\frac{M_{e} m+M_{w} w}{\rho^{2}} \frac{\partial \rho}{\partial m}=\frac{1}{\rho}\left(M_{e}-V \frac{\partial \rho}{\partial m}\right)
$$

Further, determining the derived function

$$
\frac{\partial \rho}{\partial m}=\frac{\partial \rho}{\partial c} \frac{\partial c}{\partial m}=\frac{\partial \rho}{\partial c}\left(\frac{1}{V}-\frac{c}{V} \frac{\partial V}{\partial m}\right)
$$

and substituting it into Eq.(22), we find

$$
v_{c}\left(\rho-\frac{\partial \rho}{\partial c}\right)=M_{e}-\frac{\partial \rho}{\partial c}(24)
$$

Using Eq. (24), we can determine the value of $v_{c}{ }^{0}$ by extrapolation data $\partial \rho / \partial c$ to the field of an infinitely diluted solution at $c \rightarrow 0$

$$
v_{c}^{0}=\frac{\left(M_{e}-\left(\frac{\partial p}{\partial c}\right)_{0}\right)}{\rho_{w}}
$$

Table 2: The partial molar volume $\left(\mathrm{cm}^{3} / \mathrm{mol}\right)$ in aqueous infinite diluted solutions at $293 \mathrm{~K}$. The extrapolation error of experimental data $[3,4]$ to the field of infinitely diluted solution is $\pm 1 \mathrm{~cm}^{3} / \mathrm{mol}$.

\begin{tabular}{|c|c|c|c|}
\hline Electrolyte & Eq.(16) & Eq.(21) & Meas. \\
\hline $\mathrm{LiOH}$ & -4 & -6.9 & -4.6 \\
\hline $\mathrm{Li}_{2} \mathrm{SO}_{4}$ & 16.2 & 11,1 & $-4,0$ \\
\hline $\mathrm{NaOH}$ & -3.8 & -7.5 & 17.1 \\
\hline $\mathrm{NaCl}$ & 17.5 & 15.8 & 24.3 \\
\hline $\mathrm{NaBr}$ & 23.8 & 22.7 & 35.3 \\
\hline $\mathrm{NaI}$ & 34.9 & 34.1 & 28.4 \\
\hline $\mathrm{NaNO}_{3}$ & 28.3 & 26.9 & 28.1 \\
\hline $\mathrm{NaHSO}_{4}$ & 28.1 & 24.3 & 2.6 \\
\hline $\mathrm{Na}_{2} \mathrm{SO}_{4}$ & 13.7 & 74 & 2 \\
\hline
\end{tabular}




\begin{tabular}{|c|c|c|c|}
\hline $\mathrm{Na}_{2} \mathrm{CO}_{3}$ & -3.1 & -7.4 & -3.3 \\
\hline $\mathrm{KOH}$ & 6.7 & 3.2 & 6.3 \\
\hline $\mathrm{K}_{2} \mathrm{SO}_{4}$ & 33.3 & 30.1 & 32.9 \\
\hline $\mathrm{MgCl}_{2}$ & 17.6 & 13.8 & 18.1 \\
\hline $\mathrm{CaCl}_{2}$ & 20.5 & 16.5 & 20.7 \\
\hline $\mathrm{Al}_{2}\left(\mathrm{SO}_{4}\right)_{3}$ & -4.6 & -22 & -3.6 \\
\hline
\end{tabular}

Figure 1 also shows the correspondence of the calculated values according to equations (16) and (24) and experimental data of partial molar volumes. Turning to the calculation of the density on mixed solutions should be noted that the Eq. (17), (18) are approximate because the initial Eq. (14), (16) are not linear. A strict solution can be obtained using as a starting Eq. (11) which turns into a linear one under the condition $\mathrm{a}_{\mathrm{w}}=$ const, since the value of $h_{i}$ according Eq. (4) depends only on the activity of the solvent. Then using the notation $\rho_{\mathrm{i}}{ }^{*}, \mathrm{z}_{\mathrm{i}}^{*}$ for binary isopiestic solutions, the inverse density of the mixed solution can be represented as a linear combination and expressed taking into account the boundary conditions as

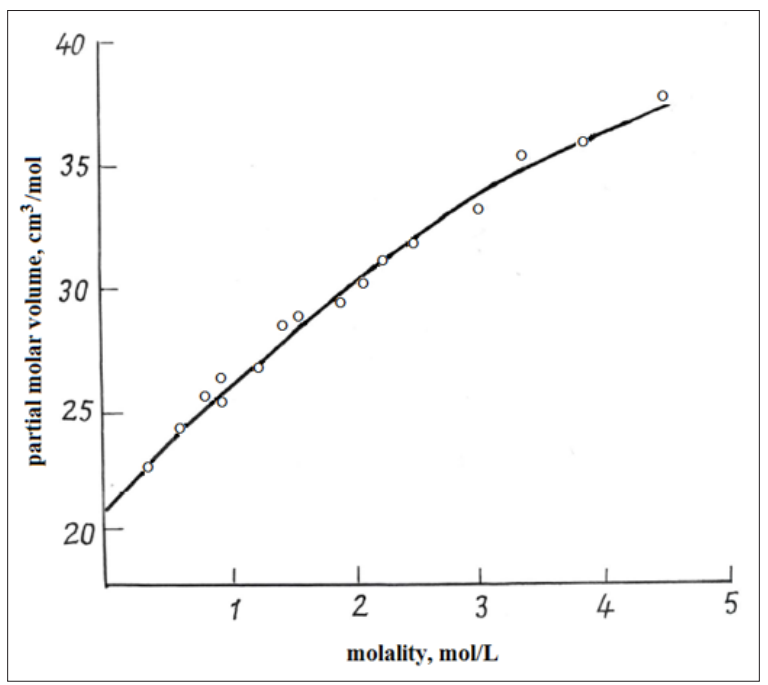

Figure 1: Partial molar volume of calcium chloride in aqueous solution at 298K. Experimental data and calculated curve.

where $\mathrm{z}_{1}$ and $\mathrm{z}_{2}$ are the concentration of components in mixed solution. However, the data in Table 3 show that the use of Eq. (17), (18) is a good approximation for calculating the density of mixed

Table 3: The density of mixed aqueous solutions $\mathrm{LiNO}_{3}+\mathrm{NaNO}_{3}$ and $\mathrm{LI}+\mathrm{KI}$ at $298 \mathrm{~K}$.

\begin{tabular}{|c|c|c|c|c|c|}
\hline & & \multicolumn{4}{|c|}{$\rho, \mathrm{g} / \mathrm{cm}^{3}$} \\
\hline \multicolumn{2}{|c|}{$c, \mathrm{~mol} / \mathrm{L}$} & \multirow{2}{*}{ Eq.(14) } & \multirow{2}{*}{ Eq.(16) } & \multirow{2}{*}{$\begin{array}{c}\text { Eq.(26) } \\
\text { Ref.[5] }\end{array}$} & \multirow{2}{*}{$\begin{array}{l}\text { Meas. } \\
\text { Ref.[5] }\end{array}$} \\
\hline $\mathrm{LiNO}_{3}$ & $\mathrm{NaNO}_{3}$ & & & & \\
\hline 8,986 & 0,688 & 1,3694 & 1,3685 & 1,3663 & 1,3656 \\
\hline 7,936 & 2,054 & 1,3968 & 1,3963 & 1,3942 & 1,3948 \\
\hline 5,463 & 3,102 & 1,3584 & 1,3633 & 1,3565 & 1,3564 \\
\hline
\end{tabular}

solutions and have significant advantage over Eq. (26), which requires a set of experimental data that is not always available. 


\begin{tabular}{|c|c|c|c|c|c|}
\hline 1,467 & 6,280 & 1,3680 & 1,3683 & 1,3705 & \\
\hline LiI & KI & & & 1,3669 \\
\hline 8,123 & 0,481 & 1,8398 & 1,8429 & 1,8437 & 1,8444 \\
\hline 5,653 & 1,238 & 1,6911 & 1,6910 & 1,6573 & 1,6916 \\
\hline 3,488 & 2,768 & 1,6589 & 1,6593 & 1,6812 & 1,6609 \\
\hline 1,603 & 4,535 & 1,6822 & 1,6823 & 1,6828 \\
\hline
\end{tabular}

\section{Conclusion}

Solvate model of solution leads to equations allowing to describe the density of electrolyte solutions with an accuracy acceptable for solving not only technological but also science problems [3-5]. The resulting equations using different concentration are convenient for the reverse calculation of the concentration reagents in the solution according to density data, which is of interest in the control of technological processes.

\section{References}

1. Allakhverdov GR (2008) Thermodynamics of electrolyte solutions. Doklady Physica 53(8): 420-424.
2. Root W (1933) The equation of density of the solutions. J Amer Chem Soc 50: 850-852.

3. Sohnel O, Novotny P (1985) Densities of aqueous solution of inorganic substances. Phys Science Data 22, Amsterdam Elsevier New York, USA.

4. Novotny P, Sohnel O (1988) Densities of binary aqueous solutions of 306 inorganic substances. J Chem Eng Data 33(1): 49-55.

5. Mikulin GI (1968) Current issues in the physical chemistry of electrolyte solutions. In: Mikulin GI (Ed.), Leningrad, Khimiya Russia.

For possible submissions Click below: 